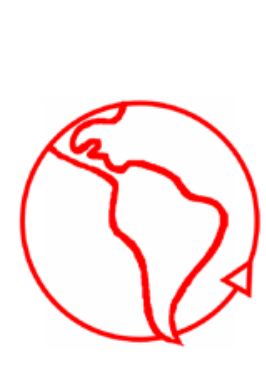

\title{
La intervención como escenario de observación de las distinciones de la teoría de la evolución
}

The intervention as a setting for observing the distinctions of the theory of evolution

Harold Dupuis Marambio

Universidad de la Frontera, Chile

Resumen

El presente texto reflexiona sobre la intervención como escenario de observación de las distinciones de la teoría de la evolución. Para ello se centra en los aportes de la perspectiva sistémica de orientación contextual como respuesta a los desafíos de hacer probable el éxito de las intervenciones en una sociedad funcionalmente diferenciada.

\begin{abstract}
This paper discusses on the intervention as a setting for observing the distinctions of the theory of evolution. This focuses on the contributions of the systemic perspective of contextual guidance in response to the challenges of making probable the success of interventions in a functionally differentiation society.
\end{abstract}

Palabras Clave: Diferenciación funcional; evolución; intervención social; reflexión; intervención sistémica

Keywords: functional differentiation, evolution, social intervention, reflection, systemic intervention

\section{Introducción}

El siguiente escrito observa el ámbito de la intervención social como escenario de aplicación de las distinciones de la teoría de la evolución socio-cultural, relevando principalmente que toda iniciativa orientada a generar transformación en una sociedad funcionalmente diferenciada tiene que resolver su propia improbabilidad de éxito, es decir, reconociendo la altísima improbabilidad de que una determinada estrategia de transformación primeramente; sea observada y aceptada por los sistemas sujetos a dicho proceso, y más aún improbable que estas iniciativas sean incorporadas como parte del propio repertorio de funcionamiento de la esferas intervenidas. La forma de ampliar la probabilidad de éxito sería observando su propia improbabilidad, desplegando esta paradoja en la elaboración reflexiva de las distinciones que dispondrá para generar el cambio.

En una sociedad funcionalmente diferenciada de esferas clausuradas en sus operaciones y abiertas a la observación de su entorno, la intervención sistémica contextual del autor Helmut Willke (1992, 1993, 2007, también Mascareño 2007) se dispone como una alternativa que está en condiciones de plantearse como estrategia que utiliza creativamente esta paradoja para ampliar las posibilidades de lograr cambios desde las determinaciones estructurales de los sistemas sujetos a los procesos de transformación. Por lo tanto, planteándose el desafío evolutivo, pero no en dirección a desestimar la 
posibilidad de la intervención, ni recaer en las premisas de la planificación en vista de sus excesivas pretensiones de determinar el futuro de los sistemas intervenidos, sino de observar la intervención como viable siempre y cuando se plantee de forma no instructiva, ni de control, sino como momento de la evolución, que no se satisface con la premisa del dejar ser (Laissez faire) evolutivo. De esta manera se plantea como una variante de la evolución que la hace reflexiva (Willke 1992).

\section{La evolución como descripción de las posibilidades de cambio}

Ante la pregunta por la posibilidad del cambio en sistemas sociales autopoiéticos, la respuesta que plantea la teoría de la sociedad de Niklas Luhmann es la evolución, como teoría que describe cómo es posible que sistemas determinados por sus estructuras transformen sus estructuras a través de sus propias operaciones (Luhmann 2007). Esta determinación estructural de los sistemas autopoiéticos indica que todo evento observado en el entorno para que ingrese como información al sistema tiene que ser seleccionado y acoplarse a las estructuras expectativas de este, de lo contrario devendrá ruido sin posibilidad de resonar en las estructuras del sistema. En estos términos, el cambio deviene auto-cambio, (Luhmann 1991), a través de la selección de las variaciones que el sistema observa como oportunidades en su entorno de reproducir su propia autopoiesis (Luhmann 2007).

La respuesta evolutiva a la posibilidad del cambio al plantearse desde las distinción sistema/entorno y la diferenciación de las funciones evolutivas de variación, selección y re- estabilización, no se limita a considerar únicamente como posibilidad de cambio lo que la sociedad desde sus autodescripciones indica como tal y sobre las que se condensan expectativas en semánticas que por lo general remiten a una crítica de la sociedad, critica que se genera como si realizara desde fuera de la sociedad. Por lo que desde la perspectiva sistémica el cambio no puede ser determinado desde fuera, desde las pretensiones que sistemas o actores tengan, sino que refieren a las propias inestabilidades que el sistema estabiliza como estado temporal y que permiten mayor sensibilidad ante las oportunidades disponibles en el entorno (Luhmann 1991: 314- 323).

La evolución observa estas semánticas del cambio más que nada como demandas que refieren a autodescripciones de los sistemas en proceso de cambio (Luhmann 1991: 321), por lo tanto, como indicaciones de una sociedad que se re- estabiliza en la posibilidad de la variación (Luhmann 2007), y en el restablecimiento de condiciones que permitan responder a las consecuencias de la diferenciación funcional en los entornos relevantes.

La evolución y su diferenciación de funciones presupone un mundo contingente donde siempre hay más posibilidades que las que se están observando en un determinado momento, por lo que, de todas las potenciales variaciones disponibles como entorno del sistema de referencia, se seleccionan las que desde la propia determinación estructural se observan como una oportunidad de auto-irritación y de transformación de sus estructuras (Luhmann 2007). Esto improbabiliza que los cambios sean producto de causas externas.

Desde estas distinciones la evolución se muestra como proceso autorreferencial y universal, en el sentido que desde la generación de una comunicación sorpresiva, inesperada, que haga una diferencia (variación) que se disponga como oportunidad de ser seleccionada y acoplada o no a las estructuras del sistema (selección) y que el sistema reorganice su unidad desde las estructuras transformadas o que las olvide (reestabilización) (Luhmann 2007). Dicho en otros términos, la diferenciación sistema y entorno y su diferencia de complejidad, genera que la variación sea una posibilidad permanente, respecto de las cuales los sistemas seleccionan el estado actual o la innovación propuesta, actualizando la reorganización de la unidad del sistema tanto en 
reafirmar el estado actual o en la innovación, de esta manera la sociedad evoluciona permanentemente.

Pero desde un punto de vista semántico y referido a las descripciones sociales de la sociedad evolución no implicaría necesariamente cambio, este concepto tendría un componente normativo que refiere a que determinados problemas que requieren determinadas soluciones más allá de las posibilidades de los afectados, o dirigir la evolución de acuerdo a los intereses de los críticos. La forma de concebir el cambio desde la idea de la crítica de la sociedad y de los actores que la practican, actualiza uno de los nudos problemáticos que la teoría de la evolución intenta resolver al desestimar como mecanismo de variación a los individuos y por lo tanto las intenciones que representen, estas concepciones de atribuir al individuo el factor de cambio, es decir, como variación evolutiva se torna confusa e inespecífica, ya que la variación evolutiva es un fenómeno tan general amplio y masivo que no podría quedar confiado a roles especiales (Luhmann 2007: 361).

Los mecanismos evolutivos refieren a la propia sociedad y no a su entorno (Luhmann 2007). En este sentido las funciones evolutivas tendrían un correlato en la propia sociedad y en su autopoiésis (variación-elementos, selección-estructuras y re-estabilizaciónsistema). De esta manera los cambios no se reducen a lo que estas semánticas de la sociedad indican, ni en la dirección que se pretende que estos tomen desde las intenciones de los generadores del cambio. Sino a comunicaciones que reproduzcan comunicaciones desviantes de las expectativas, esto lleva a poner mayor atención en el proceso de la intervención como coordinación de selectividades contingentes, que en los actores que los lleven a cabo.

Las semánticas del cambio de la sociedad como unidad esconden supuestos de comprensión de la sociedad desde la perspectiva del control y por lo tanto actualizan la referencia a una instancia central sea estructural o semántica que dirigiría de mejor manera la orientación de la sociedad, sean el mercado, el estado, el derecho o incluso la sociedad civil. Al respecto en una sociedad funcionalmente diferenciada habría que desconfiar de alguna instancia que asuma de manera generalizada la posibilidad del cambio y de la dirección de la sociedad (Willke 1992).

La evolución más que plantearse como una guía para la generación de los cambios que la sociedad espera, se plantea como observación de segundo orden respecto de cómo la sociedad describe sus propios cambios. La semántica del cambio, con toda su carga de referir la variación a los individuos, grupos o a instancias centrales que dirijan el rumbo de la sociedad, serían más que nada observaciones desde un nivel de primer orden con un alto grado de pretensión, que al ser comunicado de manera explícita se hace altamente improbable que devenga información para los sistemas afectados. La evolución como transformación de unas bajas probabilidades de surgimiento en altas probabilidades de preservación (Luhmann 2007: 326), pondría como centro de la posibilidad del cambio a la diferenciación de las funciones, y toda conexión entre la variación con la selección sea producto de la casualidad (p. 335). De acuerdo al autor, se comprendería como el punto ciego de la forma variación/selección/re-estabilización, sería la negación de que un lado de la forma diga algo directamente del otro (p. 336). La casualidad se describe como la capacidad de un sistema para usar eventos que no pueden ser producidos ni coordinados por el propio sistema; de esta manera son peligros, oportunidades, ocasiones. Sacar efectos estructurantes de las casualidades, amplifica la capacidad de elaboración de información, comparativamente con la estrechez de las estructuras existentes (Luhmann 2007).

En un contexto de complejidad moderna las esferas de sentido reaccionan alérgicamente a las instancias que intenten dirigirlos desde fuera de sus posibilidades y expectativas, esto 
mas bien, reafirma la clausura operativa con el riesgo que la apertura cognitiva devenga a su vez clausura expresada en la forma de no querer aprehender (Mascareño, 2007). Al respecto la diferenciación funcional al reafirmar la diferenciación de las funciones evolutivas releva que tanto variación como selección y re- estabilización operan de manera autónoma y diferenciada, sin que exista conexión entre ellas de manera predeterminada. La variación no se comunica como orientada a la selección, esto provocaría un alto riesgo de decepción, la realidad es conservadora - no rechaza lo que ha aceptado como idóneo (Luhmann, 2007: 396).

La evolución al desplegar la paradoja de la probabilidad de lo improbable interpela a las instancias que se orientan hacia la generación del cambio, a asumir la improbabilidad de éxito de una estrategia de este tipo, debido precisamente a que la diferenciación de las funciones evolutivas dispone a las intervenciones como variaciones mas dentro de la amplia gama de variaciones existentes y potenciales. De esta manera, la evolución puede ser observada como transformación no planificada (Luhmann 2007: 325-340) pero también como no-intervención (Willke, 1992).

\section{La planificación como coordinación de la variación y la selección}

La planificación deviene altamente improbable debido a su pretensión de que pese a que se orienta a planificar las posibilidades futuras del sistema planificado, no puede determinar en qué estado el sistema va a parar por efecto de la planificación (Luhmann 2007, Willke 1992). De esta manera, se plantea como fórmula para la modelación del futuro, que en una sociedad compleja deviene inmanejable en vista de la diferenciación de las perspectivas y de los tiempos sistémicos. No es posible saber cual serán los resultados futuros de las acciones presentes, la planificación controla los cambios por una proyección normativa de los estados futuros del sistema (Willke 1992). La sociedad se estabiliza en la variación, lo que reduce a la planificación a una variación entre una infinidad de otras posibles y la anula como anticipación de un estado deseado (Mascareño 2006).

Esta estrategia de intervención impondría sus distinciones para determinar la contingencia de los sistemas intervenidos, condicionando a que estos generen determinadas respuestas de a cuerdo a lo predeterminado por el proceso en función del cumplimiento de las metas y objetivos. Dicho en otros términos, se impondrían distinciones para coordinar la variación con la selección (Luhmann 2007: 325-340), condicionando las posibilidades selectivas del sistema intervenido.

La planificación consistiría en la ordenación de la sociedad de manera jerárquica a través de la intervención de un sistema funcional (estado, economía, derecho). De esta manera la planificación como estrategia de intervención deviene problemática y altamente improbable en sus posibilidades de éxito, al no diferenciar las funciones evolutivas conlleva a que se estabilicen formas de relacionamiento centrado en la heteronomía y limitación de la autonomía de las instancias sujetas a dicha planificación.

En síntesis, las problemáticas a las que la planificación se ve interpelada desde la evolución refieren a que en su afán por determinar los futuros posibles reduce la reestabilización del sistema a la observación de los estados que confirman el logro de lo planificado, sin observar las posibilidades propias de indeterminación y contingencia de los estados del sistema. Esto lleva a que esta fórmula reafirme la clausura de los intervenidos, al ser observada como una variación más entre otras sin posibilidad de conectar con sus posibilidades. Las formas de la planificación se hacen visibles en los diseños de políticas públicas que desde la orientación hacia la proyectización orientada al cumplimiento de metas invisibilizan las posibilidades de los afectados. 
A si como la evolución se plantea como transformación no planificada, también resta probabilidades a la intervención por lo menos en las formas clásicas de orientación directiva que se plantean desde una perspectiva externa a los sistemas sujetos a dicha iniciativas.

Considerando lo anterior, qué respuesta se podría plantear desde la teoría de la evolución respecto de las demandas urgentes de cambios que se tematizan en la sociedad, y que por lo general se realizan desde las instancias afectadas por las consecuencias de la diferenciación funcional, en este sentido, desde la perspectiva de los individuos como afectados por las consecuencias de la clausura operativa de los sistema (Mascareño 2006).

La respuesta que daría respecto del cambio desde la perspectiva normativa de las demandas acuciantes que movilizan a la intervención, sería apelar al carácter incremental, es decir, a seguir intentándolo, a generar comunicaciones de negación de las expectativas del sistema que se pretende irritar para que se produzca el cambio que se pretende generar. Seguir intentándolo hasta que de resultado, ya que un no mas otro no que se conectan y que se amplifican como posibilidad de ser seleccionados, o que cuando menos, el sistema sujeto a la irritación en la permanente re- estabilización de su estado actual, reafirme la contingencia de este estado. Este proceso se generaría por que las variaciones recientes se acoplan a las anteriores que sin haber transformado la estructura actualizaron las estructuras selectivas de ese momento, haciéndolas contingentes en el acto de seleccionar el estado actual y no la innovación (Luhmann 2007). Las variaciones de la estructura no se producen como eventos aislados, sino como conexión de eventos comunicativos que en su conexión amplifican sus posibilidades de resonancia en las estructuras del sistema, dicho de manera coloquial la recomendación es ser porfiado.

No obstante la argumentación lógica implacable de la teoría de la evolución respecto de la transformación de los sistemas en el tiempo, lo que contienen a su vez las semánticas del cambio social es una condensación de expectativas que no refieren a las observaciones de los sistemas funcionales sino a la perspectiva de los afectados por la consecuencias de la diferenciación funcional y de la clausura operativa de los sistemas funcionales (Mascareño 2006), en esta semántica se actualizan las expectativas de los individuos, la perspectiva interna de estos. Es en referencia a estas expectativas que se activan las demandas por transformación y las posibilidades de atender a las transformaciones que requieren respuestas que la evolución no podría entregar. Desde este punto se podría considerar a la evolución como sub-optima porque no permite una reacción adecuada a riesgos y situaciones de peligro de largo plazo (Willke citado en Mascareño 2006). La evolución demoraría demasiado ante las demandas normativas y que refieren a la perspectiva de los individuos.

Entonces, de qué forma se pueden configurar estrategias de intervención que fortalezcan la diferenciación de las funciones evolutivas, pero que a su vez se dispongan como una alternativa viable empíricamente de responder a las demandas emergentes de transformación en tiempos que refieran a las expectativas de cambio de los afectados. De esta manera planteándose como estrategias contra- evolutivas, o como una variante de la evolución, con una orientación reflexiva.

\section{La intervención sistémica contextual como variante de la evolución}

Las respuestas ante las demandas formuladas desde las perspectivas de los individuos por lo antes señalado no sería la planificación por su altas pretensiones de determinación las posibilidades futuras de los sistemas, y sus consecuencias en re-estabilizar un orden jerárquico de relaciones centrado en el control, ni la evolución por su lentitud y principio del dejar ser que la vuelve negación de la posibilidad de la intervención. No obstante lo anterior, en la propia evolución se encuentran las premisas centrales para la formulación 
de alternativas que, por un lado, se reconozcan como la posibilidad de guiar la evolución de manera de fortalecer la diferenciación de las funciones evolutivas, y por lo tanto la diferenciación funcional, y por otro, que se observen como una variante de la evolución, que implique una alternativa a su autorreferencia y ceguera.

La perspectiva de la intervención contextual se plantea como una formulación que por un lado, civiliza a la autorreferencia haciendo reflexiva la evolución, y también al poder planteándose como una alternativa a este, a través de una orientación no directiva ni de control (Willke 1993).

En este plano, la perspectiva se dejaría interpelar por la evolución sobre, si se pretende una transformación a través de una intervención que tenga algún grado de éxito, no tendrían que limitarse a observar como indicador de rendimiento solo el cumplimiento de las metas (primer orden) planificación, objetivos de acuerdo a las expectativas de la intervención, sino que estas puedan ser re-estabilizadas desde las posibilidades operativas de los afectados (segundo orden), por lo tanto, construirse desde la observación de la observación de las premisas estructurales y semánticas de las esferas intervenidas. Es por ello que, como principal desafío sería lograr que las acciones emprendidas puedan ser re-estabilizadas por los afectados en la forma de (auto) intervención dentro de su propio modo de operación con posibilidades de sostenimiento en el tiempo interno, dentro su semántica y formas de coordinación (Willke 1993).

Una intervención sistémica al constituirse como respuesta a las posibilidades de transformación con cierta probabilidad de éxito en una sociedad funcionalmente diferenciada, participa a su vez de la reproducción de la propia forma de diferenciación funcional, desplegándose como un mecanismo funcional a la evolución sociocultural. De esta manera, pondría en operación la diferenciación de las funciones evolutivas ubicándose como uno de ellos sin pretensiones de controlar las posibilidades de transformación.

Una estrategia de este tipo tendría que plantearse como una variación reflexiva desde la observación y reconocimiento de las perspectivas y modos de operación de los sistemas intervenidos, disponiéndose como evento en el entorno de los sistemas a regular, teniendo un potencial de variar las estructuras a las cuales apunta de manera de ser seleccionadas de forma positiva e incorporadas dentro de los respectivos modos operativos (Willke 1993). Esta variación refiere a la introducción de una interpuntuación dentro del ciclo operativo del sistema para que siga tocando su melodía pero en otro ritmo (Willke 1993)

Esta perspectiva tiene como supuesto, que todo proceso de intervención realizado en la sociedad compleja, diferenciada funcionalmente, para que tenga viabilidad de generar transformaciones que se estabilicen en las estructuras de los sistemas intervenidos, no puede plantearse como una intervención directa externa a las perspectivas de estos (Mascareño, 2007). Sino que debe construirse como un proceso reflexivo de observación de segundo orden que permita observar, distinguir e indicar las distinciones de los sistemas sujetos a intervención, e instalar las propuestas dentro de su propio contexto de operaciones (Mascareño, 2007).

El espacio dentro del que se mueve la intervención para desplegar sus acciones con sentido para el sistema intervenido, es el contexto, que refiere al marco de referencia y condiciones dentro del cual los sistemas sociales orientan sus operaciones (Mascareño 2007). Este concepto parte de la premisa de que los sistemas en su orientación al entorno observan las variantes posibles seleccionando preferentemente la información contextual, por lo tanto, aquella que posee un potencial de estructura para la delimitación de sus posibilidades operativas y pragmáticas. 
De esta manera, al no ser directa la intervención lo que se propone es el manejo del contexto del sistema sujeto a intervención, generando cambios en su entorno, e invitando a que este reaccione ante los cambios propuestos (Willke 2007). El sistema refiere a la información contextual, por lo que una intervención que se movilice en referencia a este adquiere mayores posibilidades de ser seleccionada, sin reintroducir esquemas que coordinen la variación con la selección. Con esto se quiere señalar que si bien la intervención contextual se despliega como orientación, por lo tanto, no como intervención directamente, se plantea como dispositivo que pretende movilizar determinados recursos semánticos que desde la observación de la observación de las distinciones del sistema intervenido, devengan variaciones con un potencial de selectividad que en su reestabilización establezcan un antes/después respecto de las posibilidades de selección y enlace de las operaciones del sistema.

Esta estrategia al suponer una dinámica de reflexividad desde el sistema interventor permite construirse en su diferenciación de los sistemas intervenidos, comunicar una autodescripción que permite confirmar esta diferenciación, y desde ahí producir las distinciones que permitirán definir su despliegue en la intervención. Esta reflexividad del proceso, tiene como desafío el generar la auto-reflexión en el sistema intervenido, de forma tal que este desde sus propias distinciones observe y procese las distinciones que se le quieren hacer ver. Desde acá el concepto de reflexión juega un papel determinante al introducirse como distinción que refiera a la posibilidad que el sistema observe las consecuencias de su acción en el entorno, seleccionando sus operaciones en dirección a esa observación (Willke 1993). Esto implica que no se niega la autorreferencialidad de los sistemas afectados desde la que orientan sus operaciones a la continuidad de sus propias operaciones, sino que se activa la posibilidad de observarse no como una instancia autocentrada sino como parte de un contexto de interconexiones en las que tanto su operar como el de los sistemas en el entorno traen consecuencias tanto para los otros como para sí mismos. Lo que se consideraría como determinante desde la reflexión y sus posibilidades de coordinar las distinciones del intervenido con las consecuencias de las acciones de intervención, es la ampliación de la heterorreferencia, ya que en ambos lados se presupone la capacidad de reflexión (alter/ego). De esta manera se incorporaría la perspectiva individual al orientarse a la posibilidad de regular las consecuencias del operar ciego de los sistemas que se hacen visibles en las constelaciones problemáticas que la sociedad describe y sobre las cuales la política desde su instrumental interventivo enfrenta, pero con limitados grados de éxito precisamente por su dificultad reflexiva de incorporación de la perspectiva de los afectados (Willke 1993).

Respecto de la probabilidad del éxito de la intervención la perspectiva contextual despliega creativamente la paradoja de la probabilidad de lo improbable, asumiendo su propia improbabilidad a través del reconocimiento de que la posibilidad del cambio no depende del interventor sino de lo que el sistema intervenido haga con ella (Mascareño 2007). O formulado de forma paradójica la intervención intentaría producir algo que no puede producir (Willke 1993). En esta formulación se observa que, en caso de que la intervención genere las condiciones para determinadas transformaciones, y que estas vayan de acuerdo a las expectativas de la intervención sería solo casualidad, por lo tanto, como no conexión de la variación a la selección ni la selección a la re- estabilización, abriendo el espacio para que el sistema determine los estados que considere aceptables en su reproducción autopoiética. Por lo que ante en fracaso de una intervención sistémica no existen razones para obligar a mantener la relación entre interventor e intervenido, solo queda cambiar la estrategia aprehendiendo del propio proceso (aciertos/ errores) para ampliar la probabilidad de éxito, siempre y cuando desde la demanda del intervenido observe la necesidad de hacerse partícipe de dicha iniciativa. 


\section{Palabras finales}

La intervención como escenario de aplicación de las distinciones de la teoría de la evolución permite observarla como un campo en construcción que se ve enfrentada a los desafíos de dar respuestas a las complejas condiciones tanto estructurales y semánticas de la sociedad en permanente evolución. Que desestima las formulaciones que en su pretensión de la transformación limitan las posibilidades de autonomía de lo transformado.

Si bien la propia evolución no aparece en el horizonte como la mejor alternativa ante la supervivencia de los sistemas sociales y sus consecuencias en los entornos individuales y medio ambientales, interpela a que las estrategias que dispongan con pretensiones transformacionales tengan en cuenta que en una sociedad funcionalmente diferenciada se hace cada vez más improbable el mantenimiento de estructuras que reproduzcan sin cuestionamiento dinámicas de control y externas a las posibilidades de los afectados.

Las perspectiva de orientación sistémica se visualiza como alternativa viable de hacerse operativa con importantes grados de éxito, eso si manteniendo su propia diferenciación respecto de las perspectivas que demandas rendimientos mas allá de lo que los afectados estarían dispuestos a aceptar. Tal como lo señalado, la paradoja evolutiva deviene un impulso importante, y por lo mismos una llamada de atención a las iniciativas que se despliegan permanentemente en la sociedad contemporánea. RM

\section{Bibliografía}

Esposito, E., Baraldi, C. \& Corsi, G. (1996). Glosario sobre la teoría social de Niklas Luhmann. México D. F.: Universidad Iberoamericana-Iteso- Anthropos.

Luhmann, N. (1991) Sistemas sociales, Lineamientos para una teoría general. México, D. F.: Anthropos- Universidad iberoamericana.

Luhmann, N. (2007). La sociedad de la sociedad. México D. F.: Herder, Universidad Iberoamericana.

Luhmann, N. y Di Georgi, R. (1998). Teoría de la sociedad. México D. F.: Universidad Iberoamericana- Triana.

Mascareño, A. (2007). Sociología de la intervención: Orientación sistémico contextual. En A. Kaulino (Ed.), Mediación y complejidad. Santiago de Chile: Universidad Diego Portales.

Mascareño, A (2006). Ética de la contingencia, por medio de medio del derecho reflexivo. En A. Stamford (Ed.), Sociología do Direito. A práctica da teoría sociologica. Sao Paulo: Lumen Juris.

Willke, H. (2007). Entrevista, Revista Vínculo, Julio, Santiago, Chile.

Willke, H. (1993). Formas de la autoorientacion de la sociedad, (Cap 4) En Teoría de sistemas de las sociedades desarrolladas: Dinámica y riesgo de la autoorganizacion social moderna: Titulo original: Sistemtheorie Entwickelter Gesellschaftlicher Selbsorganisation. (Manuscrito, traducción de Aldo Mascareño).

Willke, H. (1992). Societal guidance through law. In G. Teubner \& A. Febbrajo (Eds.), State, Law and Economy as autopoietic systems (pp. 354- 387). Milan: Giuffre. 
Sobre el autor

Harold Dupuis obtuvo su grado de Magister en Antropología y Desarrollo en la Universidad de chile y su grado de Sociólogo en la Universidad de la Frontera.

haroldupuis1685@yahoo.com

\section{Contacto}

Sin dirección postal

Recibido: Julio/2011

Aceptado: Agosto/2011

Artículo disponible en: http:// www.facso.uchile.cl/publicaciones/mad/25/ dupuis02.pdf 